\title{
A Review of Various Histogram Equalization Techniques for Image Enhancement
}

\author{
MD Afaque Azam \\ School of Electronics Engineering, Kalinga Institute of Industrial Technology
}

\begin{abstract}
An image is a collection of pixels arranged in a meaningful fashion. The brightness of these pixels are represented by intensity levels which is a proportionate number stored in computer memory. In general the more the intensity of pixels is uniformly distributed the better the image quality. However sometimes due to various sudden, unavoidable or intentional changes in lighting condition the object or the scene under consideration is not properly exposed. In such cases we resort to image processing techniques to enhance the image quality. In subsequent sections we introduce histogram, different equalization techniques and their simulation.
\end{abstract}

Keywords: Image, processing, contrast, histogram, simulation

\section{Introduction}

An image in real world is a three dimensional object. When projected on transducers we get a two dimensional discrete representation of the real object. If we look closely on this image it is formed of small pixels whose number depend upon the size and resolution of the camera. The quality of an image is subjective to viewers. Overall the quality of an image depends upon lighting conditions, exposure, object motion, handshake etc. If this image is not captured properly then the quality of the pictures degrades. With the advent of digital computers and introduction of new processing al-gorithms it is now possible to enhance the image quality. However the technique depends largely on the application at hand. One such image enhancement technique is histogram equalisation.

\section{Histogram Equalisation Methods}

An image consists of a very large number of pixels. Each pixel in the image has a particular discrete intensity in the range $0-255$ for eight bit registers. This intensity range is broken into 256 bins start-ing from 0 to 255. Then the entire image is scanned and the bin corresponding to the intensity of the pixel, is increased by one each time a pixel with that intensity is met. In this way we get a histogram of the intensity of pixels in the image.

If $\mathrm{MxN}$ is the size of image and $\mathrm{b}_{\mathrm{i}}$ is the intensity of the $\mathrm{i}^{\text {th }}$ pixel then histogram count of the $l$ th $\operatorname{bin} \boldsymbol{h}(l)$ $\boldsymbol{h}(l)=\boldsymbol{h}(l)+1$ If $b(i)=\boldsymbol{h}(l)$ for $l$ in $[0,1, \ldots, 255]$.

Also $\sum_{l=0}^{255} \mathbf{h}(1)=M N$.

Hence $\frac{h(l)}{\sum_{7=n}^{255} h(l)}$ gives the probability of occurrence of $l^{\text {th }}$ level in the given image denoted by $\mathrm{p}(l)$.

One such histogram is shown in one of the figures.

Having obtained the probability density function (pdf) of the intensity levels in the image we can construct its cumulative distribution function $C(L)=\sum_{i=0}^{L} p(i)$.

One such cumulative distribution function scaled in the range 0-255 is shown in one of the figures in the result section.

However if the object is not properly exposed to light then bins of lower intensity will have large number of pixels causing an uneven distribution of intensity. This poor distribution of intensity among pixels causes the image quality to degrade. The next step in histogram equalisation is to change the distribution of pixels in original histogram to a uniformly distributed histogram so that all the brightness levels are equally distributed. For this transformation $\mathrm{T}$ of a pixel intensity $l$ to a uniform distribution:

$T(l)=l_{0}+\left(l_{255}-l_{0}\right) * \sum_{i=0}^{l} p(i)$ 
The resulting histogram has more or less a uniform distribution of pixels across all intensity levels. One such equalised histogram is shown in the figures provided below. However meanwhile in the process above the mean brightness level of the image is shifted near to the mid of the intensity range. Due to this the image loses its original brightness level. In order to preserve the mean brightness level of the image another equalisation technique called Bi-level histogram equalisation method is used. In this technique the whole histogram of original image is divided into two parts the boundary lying at the mean intensity of the original image. Then equalisation is performed on the two halves separately. This preserves the mean intensity of the result image because the number of pixels on both sides of the boundary intensity level is still the same after processing. In terms of equations the processing can be explained as follows:

If $l_{\mathrm{m}}$ is the mean intensity level of the image. Then for lower half intensity image the transformation applied will be:

$T(l)=\operatorname{lm} * \sum_{i=0}^{l} p(i)$

And for the upper half of the intensity level image following transformation is applied;

$T(l)=(l m+1)+\left(l_{255}-l_{\mathrm{m}}-1\right) * \sum_{i=0}^{l} p(i)$

The two equalised histograms are then joined together and a composite histogram is formed. The resulting equalised image and its histogram is shown in the figures provided in the result section.

\section{Minimum Mean Brightness Level:}

In this method the aim is to minimise the error between the mean intensity levels of the original histogram and the mean of those histograms which are equalised after varying the threshold intensity before equalisation. Finally that histogram is selected which has minimum error. For this the histogram of the original image is divided into two parts whose threshold intensity changes with each iteration. Then the two histograms are independently equalised before joining them and the associated error is calculated. On doing some mathematics we obtain a recursive solution to find the mean brightness error given as:

$\mathrm{E}_{\mathrm{T}+1}(\mathrm{Y})=\mathrm{E}_{\mathrm{T}}(\mathrm{Y})+\frac{1}{2}\left[1-l_{255} * \mathrm{P}\left(\mathrm{X}_{\mathrm{T}+1}\right)\right]$

Once the matrix of error has been formed the threshold level with minimum error is selected and then the procedure similar to $\mathrm{Bi}$ level histogram equalisation is followed. The results of this histogram equalisation technique is shown in the figures in the result section.

\section{Weighted Bi Level Histogram Method:}

In this method the whole histogram is segmented into different regions by locating the valleys in the histogram. Then each region is separately equalised. When combining different regions of the histogram together weights are given to individual segments depending upon the density of the segment such that overall when weights are added they sum up to one. The following diagrams shows the process as just described.
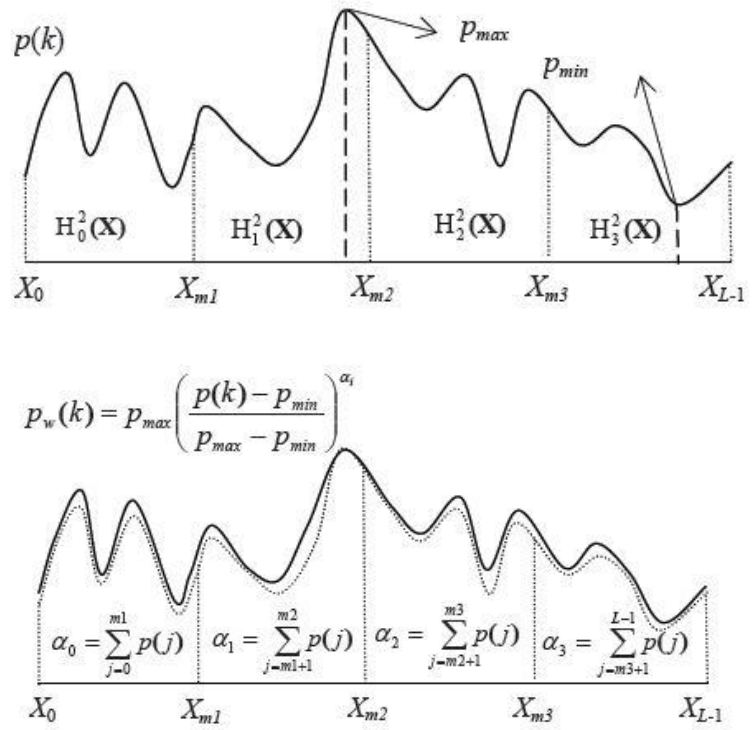

Fig 1. [3] A sample histogram showing peak and valley regions. Below is shown the segmented regions of histogram given different weights. 


\section{Result}

We have implemented all the above algorithms for the techniques discussed above on MATLAB. The result of the simulation is shown below. We have used Baboon as the test image for performing simulations and testing results. As can be seen in the results we have shown first the original image in figure 2:

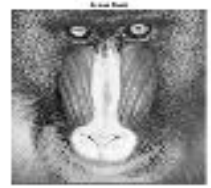

(3)

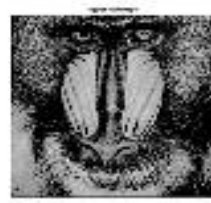

is

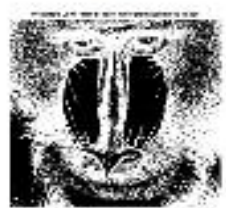

$(a)$

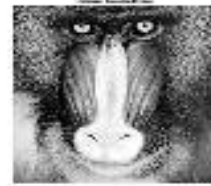

(2)

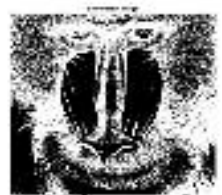

is

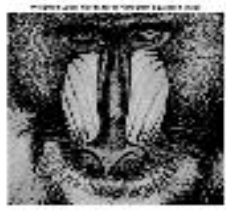

(it)

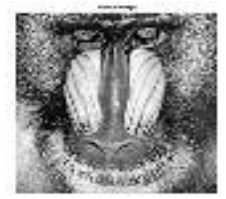

(i)

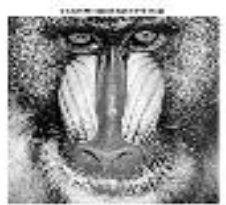

is)

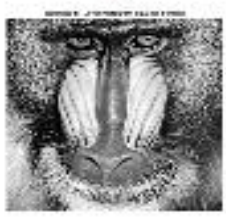

(6)

Fig 2. (a) Original Image of Baboon (b) Image after histogram equalisation (c) Output image after Minimum Mean Brightness Error histogram equalisation (d) Upper half image (e) Lower half image (f) Output image of Bi- level histogram equalisation (g) Lower half image of WBLHE technique (h) Upper half image of WBLHE technique (i) Output equalised image of Weighted Bi level Histogram equalisation.

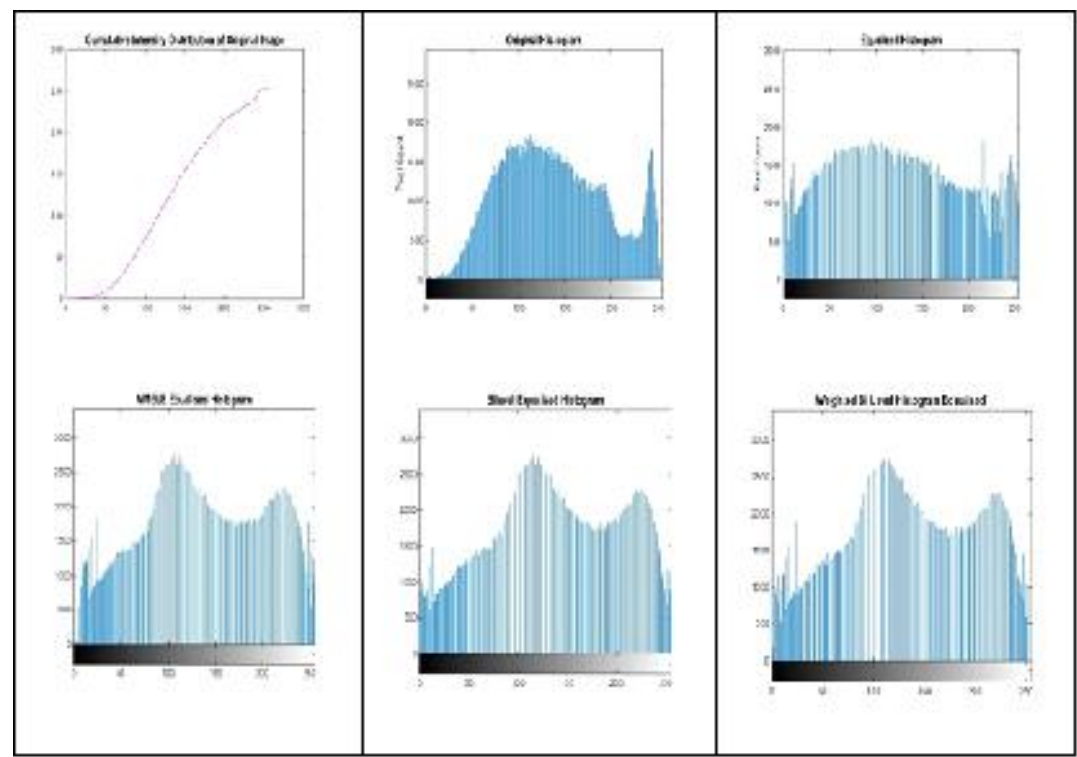

Fig 3. Right from top left (a) Cumulative intensity histogram (b) Original histogram of image baboon (c) Equalised histogram using normal HE technique (d) MMBLE histogram (e) BLHE histogram (f) WBLHE histogram

\begin{tabular}{|l|l|l|}
\hline Equalisation Method & PSNR & MSE \\
\hline Histogram Equalisation(HE) & 13.6587 & 135.1067 \\
\hline Bi-Level Histogram Equalisation(BLHE) & 18.0836 & 97.7640 \\
\hline Weighted BLHE & 18.3120 & 105.0501 \\
\hline Minimum Mean Brightness Level (MMBLE) & 18.4114 & 110.3757 \\
\hline
\end{tabular}

Table 1. PSNR and MSE performance comparison for various equalisation techniques. 


\section{Conclusion}

In this paper we reviewed major histogram equalisation techniques. Then we checked their performance on the basis of different metrics like PSNR and MSE. We found that Minimum Mean Brightness Level gives better performance in terms of PSNR while Bi-level histogram equalisation technique is better when compared in terms of MSE (Mean Square Error). In future work we would like to extend the above discussed ideas to propose a new better method for histogram equalisation.

\section{References}

[1]. "Improvement of histogram equalization using bi-histogram equalization technique for image enhancement". Sarthak Patnaik, Rasmita Lenka, Sidhartha Sinha

[2]. Chen, Soong-Der, and Abd Rahman Ramli. "Minimum mean brightness error bi-histogram equalization in contrast enhancement." Consumer Electronics, IEEE Trans-actions on 49.4 (2003): 1310-1319.

[3]. Kim, Mary, and Min Gyo Chung. "Recursively separated and weighted histogram equal-ization for brightness preservation and contrast enhancement." Consumer Electronics, IEEE Transactions on 54.3 (2008): 1389-1397.

[4]. Gonzalez, Rafael C. "RE woods, Digital Image Processing." Addison-Wesely Publishing Company (1992). 